\title{
Wettability of mineral surfaces by water and carbon dioxide
}

\author{
EMILY WEI-HSIN SUN,' IAN C. BOURG ${ }^{2}$
}

Civil and Environmental Engineering, Princeton University, Princeton, New Jersey, United States esun@princeton.edu 2Civil and Environmental Engineering, Princeton University, Princeton, New Jersey, Unites States bourg@princeton.edu

The wettability of mineral surfaces by water versus supercritical carbon dioxide $\left(\mathrm{scCO}_{2}\right)$ is one of the most important phenomena controlling the migration of $\mathrm{ScCO}_{2}$ in porous sedimentary rocks in carbon mitigation technologies including CCS, BECCS, and CO2-EOR. In particular, the contact angle formed by water and $\mathrm{scCO}_{2}$ with the surfaces of mineral grains determines the efficiency of important trapping mechanisms: stratigraphic trapping and residual trapping. This contact angle remains poorly understood in conditions relevant to geologic carbon storage, in large part because values reported in different studies vary widely, making it difficult to confidently predict multiphase flow patterns and their sensitivity to temperature, pressure, salinity, and organic residues during $\mathrm{CO}_{2}$ injection in sedimentary rocks.

In this paper, atomistic simulations are employed to circumvent experimental challenges (such as surface contamination and critical conditions) and answer recurrent questions posed by studies of small droplets meant to inform larger models (such as the validity of the Young and YoungLaplace equations at very small scales). Molecular dynamics (MD) simulations have shown significant promise as a complementary technique to elucidate interfacial tensions and contact angles at the nanoscale, and their trends with respect to changes in temperature and pressure. We build upon the MD simulation methodology used in previous studies to include three significant advances. First, we use interatomic potential parameters previously tested against experimental data on silica-water and $\mathrm{CO}_{2}$-water interfacial properties, and we carry out extensive tests to determine the sensitivity of these parameters. Second, we carry out relatively long simulations and use a variety of methods to ensure our systems reach a fully equilibrated state before predicting any properties. Third, we calculate the local stress distributions in a manner that provides new atomistic level insight into wetting and capillary forces in detail. Thus, we are able to probe the sources of variability of previous experimental measurements and computational predictions, as well as the scale of measurement and validity of macroscopic principles near the solid surface. 